\title{
Seguridad alimentaria y nutricional, derecho humano a la alimentación adecuada y soberanía alimentaria: una aproximación desde los instrumentos del derecho internacional
}

\section{Food and nutrition security, human right to adequate food and food sovereignty: an approximation from the instruments of international law}

\section{Adolfo Martín Hurtado Díaz}

Consultor e Investigador Independiente, ORCID: https://orcid.org/0000-0003-0238-6405, adolfohurtado@consultant.com

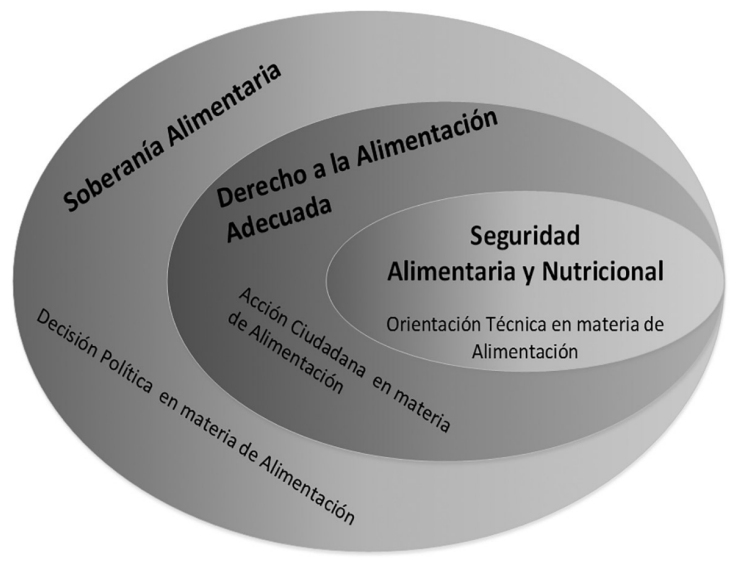

\section{RESUMEN}

La seguridad alimentaria y nutricional, derecho a la alimentación y soberanía alimentaria son tres abordajes correlacionados fundamentales para el tratamiento y adecuada solución de los asuntos alimentarios de nuestras sociedades. Estos tres conceptos abordan la materia alimentaria desde tres ángulos que entre si son complementarios. Las debilidades en la comprensión de estos tres abordajes han generado dificultades de diálogo entre los diversos actores, nacionales e internacionales, relacionados a esta materia.

Palabras clave: Centroamérica, alimentos inocuos, acceso físico, acceso económico y acceso social.

\section{ABSTRACT}

Food and nutrition security, right to food and food sovereignty are three fundamental correlated approaches for the treatment and adequate solution of the food issues of our societies. These three concepts deal with food from three angles that are complementary to each other. The weaknesses in the understanding of these three approaches have generated difficulties of dialogue between the various national and international actors related to this matter. Keywords: Central America, safe food, physical access, economic access and social access.

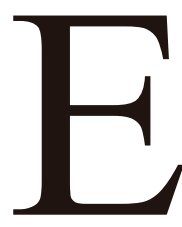

n la actualidad, en materia alimentaria, tenemos tres conceptos fuertes que organizan las acciones públicas y privadas que no tratan exactamente de lo mismo, pero sí de elementos comunes. Estos son Seguridad Alimentaria y Nutricional (SAN), Derecho Humano a la Alimentación Adecuada (DHAA) y Soberanía Alimentaria (SobAL). El marco epistemológico de los gobiernos de la región y de los organismos internacionales está orientado al derecho a la alimentación adecuada y seguridad alimentaria y nutricional. En cambio, las organizaciones sociales de la región principalmente abordan la problemática desde la soberanía alimentaria. El tratamiento de esta materia se da como si estos abordajes fueran temas excluyentes, o trataran naturalezas distintas.

En este escrito se pretende una rápida aproximación a la naturaleza e interrelaciones de estos tres conceptos. Ocupamos los instrumentos internacionales en materia de DHAA $\mathrm{y}$ las declaraciones internacionales en materia de SobAL.
Recibido: 4 de abril del 2019

Aceptado: 28 de mayo del 2019

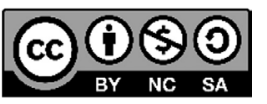

C Copyright 2019. Universidad Nacional Agraria
Los artículos de la revista La Calera de la Universidad Nacional Agraria, Nicaragua, se comparten bajo términos de la licencia Creative Commons: Reconocimiento, No Comercial, Compartir Igual. Las autorizaciones adicionales a las aquí delimitadas se pueden obtener en el correo edgardo.jimenez@ci.una.edu.ni 
Esta reflexión se desarrolló en el marco de la Alianza por el Derecho Humano a la Alimentación de Centroamérica para la focalización de la incidencia en las políticas regionales agroalimentaria en el marco de los seis estudios del Estado de la Soberanía Alimentaria de Centroamérica. Esta síntesis fue desarrollada en el marco de la investigación de proyectos relacionados con el "desarrollo de la plataforma PECOSOL-CONSUACCIÓN para la SAN en Centroamérica”. OIKOS - Cooperação e Desenvolvimento.

Seguridad alimentaria y nutricional. La $39^{\text {ava }}$ sesión del comité de seguridad alimentaria mundial (CFS), en el documento "en buenos términos con la terminología", en sus conclusiones señala que: "existe seguridad alimentaria y nutricional cuando todas las personas tienen en todo momento acceso físico, social y económico a alimentos inocuos, cuyo consumo es suficiente en términos de cantidad y calidad para satisfacer sus necesidades y preferencias alimentarias, y se sustenta en un marco de saneamiento, servicios sanitarios y cuidados adecuados que les permiten llevar una vida activa y sana".

La primera oración del párrafo seis de la Observación General \#12 (OG 12) del Pacto Internacional de los Derechos Económicos Sociales y Culturales (PIDESC) señala que: "el derecho a la alimentación adecuada se ejerce cuando todo hombre, mujer o niño, ya sea sólo o en común con otros, tiene acceso físico y económico, en todo momento, a la alimentación adecuada o a medios para obtenerla".

Se entiende que la SAN es condición necesaria para alcanzar la promoción del DHAA. En las definiciones de adecuación y sostenibilidad en sus diversos párrafos establece la necesidad de garantizar la cantidad y la diversidad. En el marco de definición de la adecuación, en la primera parte del párrafo nueve expresa:

“... que el régimen de alimentación en conjunto aporta una combinación de productos nutritivos para el crecimiento físico y mental, el desarrollo y el mantenimiento, y la actividad física que sea suficiente para satisfacer las necesidades fisiológicas humanas en todas las etapas del ciclo vital, y según el sexo y la ocupación" (OG 12, 1999, p 3, prf 9).

SAN, es un concepto técnico vinculado a asuntos relacionados a la alimentación humana, y como señala en el párrafo 32 del documento citado de la CFS, hace hincapié en que el objetivo general es lograr a la vez la "seguridad alimentaria" y la "seguridad nutricional" como una meta única y unitaria de las medidas de las políticas y los programas. También se argumenta que esta formulación alienta a las diferentes comunidades la práctica de integrar mejor su trabajo con vistas al logro de los objetivos de la seguridad alimentaria y la seguridad nutricional". Tenemos entonces: que SA + $\mathrm{SN}=\mathrm{SAN}$, y $\mathrm{SAN}>\mathrm{SA}$; $\mathrm{SN}$.

La SAN se orienta a establecer el perfil, en materia alimentaria, de las capacidades que las personas y las sociedades deben de considerar para tener la base para la promo- ción del DHAA, por lo que podemos señalar que la SAN es de orientación técnica. En el párrafo 25 de la OG 12 se establecen los instrumentos de las estrategias nacionales para la promoción del DHAA y los derechos vinculados, y con ello, el marco institucional / sectorial desde el que se promueve:

"la estrategia se ocupará de todas las cuestiones y medidas críticas relativas a todos los aspectos del sistema alimentario, en particular la producción, elaboración, distribución, comercialización y consumo de alimentos sanos, así como las medidas paralelas en materia de salud, educación, empleo y seguridad social. Hay que procurar gestionar y utilizar de modo más sostenible los recursos alimentarios naturales y de otro tipo en los niveles nacional, regional, local y doméstico" (OG 12, 199. P 6, prf 25).

Derecho a la alimentación adecuada. Es un concepto vinculado a derechos humanos (DDHH), en el marco de los derechos económicos, sociales y culturales. En el enfoque de $\mathrm{DDHH}$, los resultados se definen en términos de derechos, y estos son tan importantes como los procesos que los hacen posibles. Los procesos deben de basarse en estándares y principios internacionales de DDHH. La implementación del enfoque de DDHH busca la realización progresiva de todos los derechos humanos a largo plazo y la mejora de las capacidades de titulares (para reclamar) y detentores (para cumplir) a medio plazo.

El párrafo 23 de la OG 12 del PIDESC establece los principios específicos al DHAA y la buena gobernanza relacionada a esta: "la formulación y aplicación de estrategias nacionales para el derecho a la alimentación exige el pleno cumplimiento de los principios de responsabilidad, transparencia, participación popular, descentralización, capacidad legislativa e independencia de la magistratura. Es esencial un buen gobierno para la realización de los derechos humanos, incluida la eliminación de la pobreza, y para asegurar medios de vida satisfactorios para todos". Tenemos entonces: SAN + Derecho $($ ciudadanía $)=$ DHAA, y DHAA $>$ SAN .

\section{Soberanía alimentaria}

Soberanía. Es un concepto vinculado a la Teoría Política y en la Doctrina del Derecho Internacional:

"en sentido amplio el concepto político jurídico de soberanía sirve para indicar el poder de mando en última instancia en una sociedad política y, por consiguiente, para diferenciar a esta de las otras asociaciones humanas, en cuya organización no existe tal poder supremo, exclusivo y no derivado" y agrega a seguir y por tanto tal concepto está estrechamente vinculado al de poder político" (Bobbio, 1986).

Soberanía alimentaria. En la Declaración "Primero está la Soberanía Alimentaria de los pueblos ¡Fuera la OMC de la agricultura y la alimentación!” de La Habana en 2001 señala que: 
"soberanía alimentaria es el derecho de cada pueblo a definir sus propias políticas agropecuarias y en materia de alimentación, a proteger y reglamentar la producción agropecuaria nacional y el mercado doméstico a fin de alcanzar metas de desarrollo sustentable, a decidir en qué medida quieren ser autodependientes, a impedir que sus mercados se vean inundados por productos excedentarios de otros países que los vuelcan al mercado internacional mediante la práctica del 'dumping', y a darle preferencia a las comunidades locales pescadoras respecto al control del uso y los derechos sobre los recursos acuáticos".

Lo anterior, expresa la Soberanía Alimentaria como una demanda política basada en derechos. Esto tiene su reflejo en los instrumentos internacionales donde se orienta a las estrategias nacionales (Políticas):

“... el Pacto especifica claramente que cada Estado Parte adoptará las medidas que sean necesarias para garantizar que todas las personas queden libres del hambre y que puedan disfrutar lo más pronto posible del derecho a una alimentación adecuada. Esto exigirá aprobar una estrategia nacional que garantice la seguridad alimentaria y de nutrición para todos, sobre la base de los principios de los derechos humanos que definen los objetivos, formular las políticas y los indicadores correspondientes. También deberán identificarse los recursos disponibles para cumplir los objetivos y la manera de aprovecharlos más eficaz en función de los costos" (OG 12, 1999, p 6, prf 21).

Así tenemos: DHAA + Soberanía $=$ Soberanía Alimentaria, y Soberanía Alimentaria $>$ DHAA $>$ SAN .

La soberanía alimentaria en el marco del derecho humano a la alimentación adecuada. Como ya se ha señalado, las demandas de inclusión en las atenciones y soluciones al derecho a la alimentación adecuada en el diseño de las estrategias nacionales SAN, se encuentra en el párrafo 23 de la OG 12 del PIDESC que señala los principios específicos del DHAA, además de los principios generales de los DDHH la "responsabilidad, transparencia, participación popular, descentralización" entre otros. Esto se ve reforzado con la comprensión específica de No Discriminación, de los DDHH, aplicados para el DHAA:

"la estrategia debe prestar una atención especial a la necesidad de prevenir la discriminación en el acceso a los alimentos o a los recursos destinados a alimentos. Esto debe incluir los siguientes elementos: garantías de un acceso completo y equitativo a los recursos económicos, especialmente para las mujeres, incluido el derecho a heredar y a poseer tierras y otros bienes, y de acceso al crédito, a los recursos naturales y a una tecnología adecuada; medidas para respetar y proteger el trabajo por cuenta propia y los trabajos remunerados de modo que aseguren una vida digna para los asalariados y sus familias (como estipula el inciso ii) del párrafo a) del artículo 7 del Pacto); mantener registros sobre los derechos a la tierra (incluidos los bosques)" OG 12, 1999, p 6, prf 26).

Lo anterior, conjuntamente con el párrafo 25 sobre las responsabilidades de las instituciones nacionales para la promoción del DHAA, guardan íntima concordancia con las demandas de los movimientos sociales en general y de Vía Campesina en particular como los derechos de las campesinas y campesinos, biodiversidad y recursos genéticos, cambio climático y agro-ecología, entre otros, señalados en los distintos informes de Vía Campesina como el Informe de la VI Conferencia Internacional de Vía Campesina.

\section{CONCLUSIONES}

Los conceptos de SAN, DHAA y soberanía alimentaria, no sólo no son elementos conceptos distintos, si no que se explican y se complementan entre sí, guiando distintos niveles de decisiones sociales en materia de los alimentos.

De ahí, que estaremos hablando de SAN, cuando estamos definiendo el estado y los determinantes de la misma referidos a la alimentación adecuada de los individuos.

Estaremos hablando de derecho a la alimentación cuando lo anterior se desarrolla en medio de procesos sociales con participación ciudadana, transparencia y resguarde los principios de la promoción de los DDHH.

Y estaremos hablando de soberanía alimentaria, cuando el conjunto de estas acciones sean resultados de decisiones soberanas de las sociedades que las toman. La sociedad civil, en general, y Vía Campesina en particular, proponen una agenda que nos lleve a un desarrollo local incluyente, integrado y sostenible.

\section{AGRADECIMIENTOS}

El autor agradece a la Fundación Calouste Gulbenkian y Camões - Instituto de la Cooperación y la Lengua IP por el financiamiento de este esfuerzo.

\section{REFERENCIAS BIBLIOGRÁFICAS}

Comité de Seguridad Alimentaria Mundial (CFS) (2012). En buenos términos con la terminología: seguridad alimentaria, seguridad nutricional seguridad alimentaria y nutrición, y seguridad alimentaria y nutricional. 39. ${ }^{\circ}$ período de sesiones; Tema V. a; Roma, Italia;

Consejo Económico y Social, Naciones Unidas; Observación General 10 del PIDESC (1998). La función de las instituciones nacionales de DDHH en la protección de los DESC. Referente al Art. 2 del PIDESC. Ginebra;

Consejo Económico y Social, Naciones Unidas; Observación General 12 del PIDESC (1999). El derecho a la alimentación adecuada. Referente al Art. 11 del PIDESC; Ginebra; 
Declaración “primero está la soberanía alimentaria de los pueblos ¡fuera la OMC de la agricultura y la alimentación!” (2001). la Habana Cuba;

Declaración Universal de los Derecho Humanos (1948). Adoptada y proclamada por la Asamblea General de Naciones Unidas;

Norberto Bobbio y Nicolau Matteucci, (1986). Diccionario de Política, Ed. S. XXI, 4ta Ed, 1986, Ciudad de México,

FAO - PRESANCA (2013). Comparación de conceptos: seguridad alimentaria, soberanía alimentaria y derecho a la alimentación"; Taller de formación de formadores, Presentación;

Hurtado Adolfo (2015). Estado de la Soberanía Alimentaria de Centroamérica. Repensando la gobernabilidad regional para unn desarrollo rural incluyente, integrado y sostenible frente a los grandes retos de la globalización; ADHCA/OIKOS/UE/UNDEF;

Lorente, Isabel (2004). El derecho a la alimentación y la soberanía Alimentaria; Trabajo Monográfico Posgrado en Agentes de Desarrollo Internacional en España, Universidad Politécnica de Catalunya (UPC);

Naciones Unidas (2003). El Desarrollo Basado en un Enfoque de los Derechos Humanos: Hacia una Comprensión Colectiva entre las Agencias de las Naciones Unidas, PNUD;

Naciones Unidas (2007). El Sistema de Tratados de Derechos Humanos de las Naciones Unidas. Introducción a los tratados fundamentales de derechos humanos y a los órganos creados en virtud de tratados; Folleto informativo No 30; Oficina del Alto Comisionado de las Naciones Unidas para los Derechos Humanos; Geneva

Pacto Internacional de los Derechos Económicos Sociales y Culturales (PIDESC) (1976).

Vía Campesina (2014). VI Conferencia Internacional de Vía Campesina; Yakarta. 